\title{
Event-Based Processing of Neutron Scattering Data
}

\author{
Peter F. Peterson, Stuart I. Campbell, Michael A. Reuter, Russell J. \\ Taylor, Janik Zikovsky \\ Neutron Data Analysis and Visualization, Oak Ridge National Laboratory, \\ Oak Ridge, TN, USA
}

\begin{abstract}
Many of the world's time-of-flight spallation neutrons sources are migrating to recording individual neutron events. This provides for new opportunities in data processing, the least of which is to filter the events based on correlating them with logs of sample environment and other ancillary equipment. This paper will describe techniques for processing neutron scattering data acquired in event mode which preserve event information all the way to a final spectrum, including any necessary corrections or normalizations. This results in smaller final uncertainties compared to traditional methods, while significantly reducing processing time and memory requirements in typical experiments. Results with traditional histogramming techniques will be shown for comparison.
\end{abstract}

Keywords: neutron, software

\section{Introduction}

The Spallation Neutron Source (SNS) at Oak Ridge National Laboratory

$3[1,2]$ measured first neutrons April 28, 2006. Besides the promised neutron

4 flux increase over existing sources and novel instrument design, the biggest 
feature advertised was event mode data acquisition. Event mode provides three pieces of information for each detected neutron: detector pixel identifier, total time-of-flight from source to detector, and wall clock time of the proton pulse the neutron is associated with. The full list of events can be stored for later processing. The time-of-flight is the time from the neutron to be emitted from the moderator until it is measured by the detector. The detector pixel identifier, or pixel id, is a unique index to the detector pixel in the instrument. This differs from the traditional data acquisition mode of measuring histograms where each detected neutron is given a pixel identifier and time-of-flight then collected into histograms, without information about the pulse the neutron is associated with.

Limited memory and bandwidth of computers when time-of-flight neutron scattering was being developed is the reason for histogram mode $[3,4,5]$. Generally the experimenter made a choice of either having more detector pixels or time-of-flight bins, balancing them as anticipated for the experiment. With event mode, the neutrons are recorded at full resolution of the data acquisition electronics. At SNS this is 100ns for time-of-flight and each individual pixel. While this new acquisition mode was available since the first neutrons were measured, techniques for processing the events had not been developed. For the first few years of operation data was reduced by first histogramming the events, then processing them using traditional techniques. Note that this is still an available option, but much of the data reduction at SNS has been converted to use the techniques described below. 


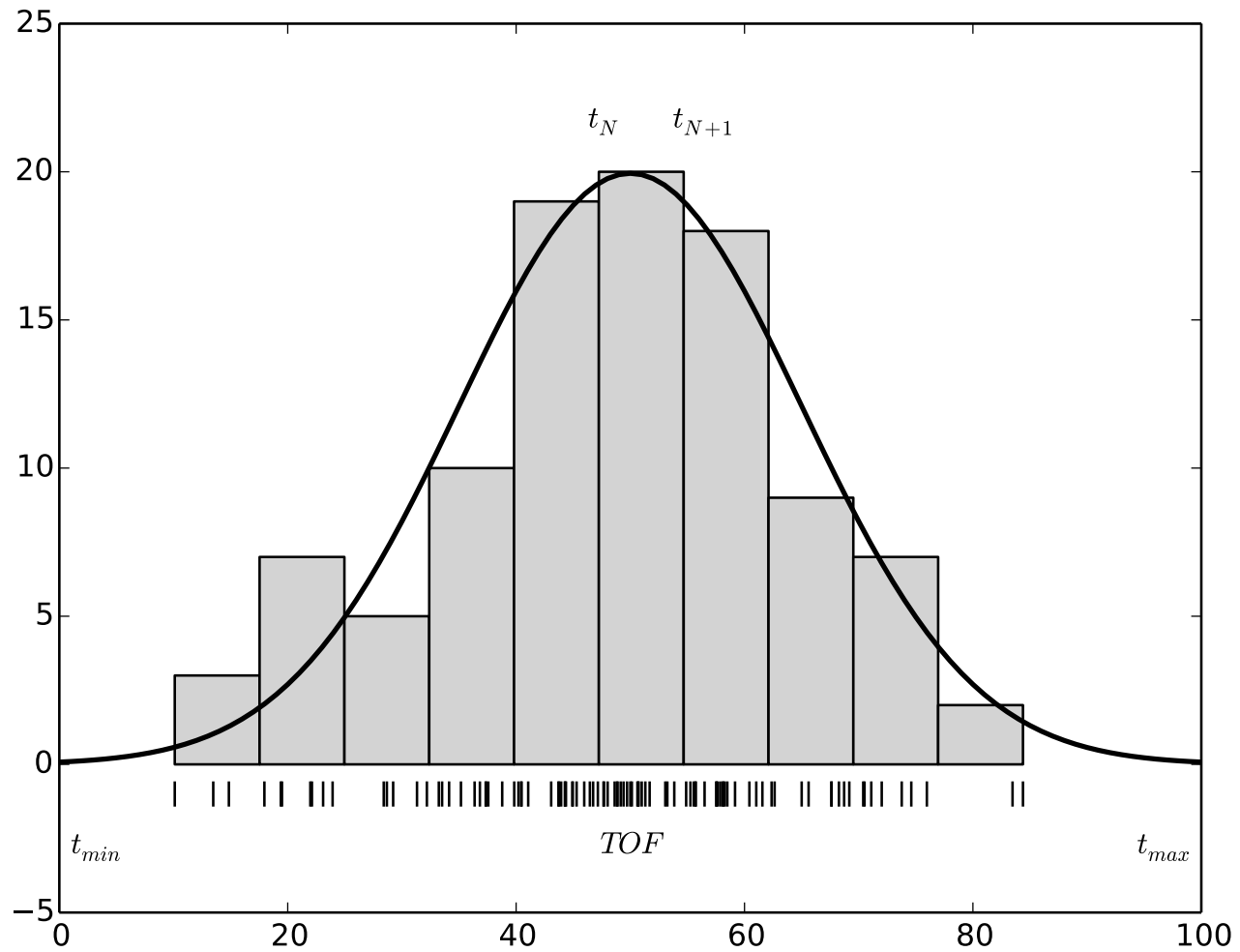

Figure 1: Schematic diagram of histogramming. Dashes below are individual events. Boxes are the histogram representation. Curved line is the normal distribution for reference. 


\section{Histogramming}

Before the SNS, most experiments on time of flight neutron sources, data was collected in what is known as Histogram Mode. What do we mean by this? Histograms divide the total range of time-of-flights $\left(t_{\min } \rightarrow t_{\max }\right)$ into a number of channels or bins. When a neutron is detected, its time-of-flight $(T O F)$ is determined and the scaler counter for the corresponding histogram channel is incremented.

The time-of-flight $(T O F)$ of a neutron recorded into the time channel $N$ can be defined as

$$
t_{\min } \leq t_{\min }+t_{N} \leq T O F<t_{\min }+t_{N+1} \leq t_{\max }
$$

where $t_{N}$ is the starting time boundary for the $N^{t h}$ histogram channel. Figure 1 illustrates this. For the $N^{\text {th }}$ histogram channel, the most likely time-of-flight for the neutron is given by

$$
T O F=t_{\min }+\frac{1}{2}\left(t_{N+1}-t_{N}\right)
$$

From this we can see that the time-of-flight accuracy of a given feature in the collected spectrum will depend on the width of the time channels that we use in the data acquisition. Furthermore, an increase in the channel width would also move the position of any feature by half the increase in channel width. For a more detailed discussion of choosing histogram bin widths can be found in [3]. 

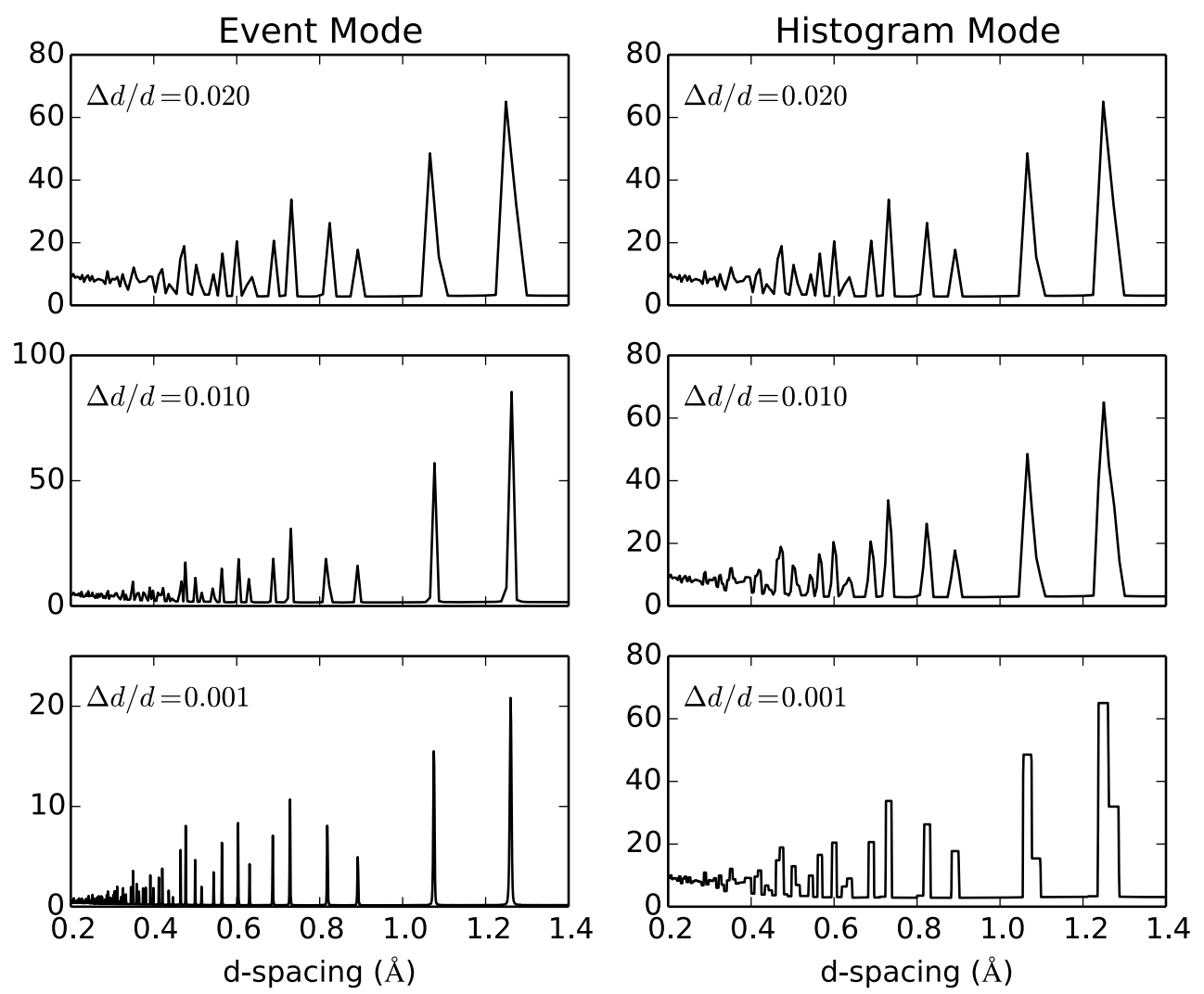

Figure 2: A comparison of the relative differences observed in diffractograms arising from the rebinning of histogram or event data. Each lower figure is a rebinned version of the one above it. All four are done with constant $\Delta T / T$ binning with the left column being the with event data and the right being historgram, for comparison. The data presented is from a Diamond sample measured on the instrument POWGEN.[6] 


\section{Dynamic Histogramming}

One great advantage of event-based processing is that histogramming can be performed dynamically, using the raw event data. In histogrammed data, the distribution of neutrons' times of flight within a single bin is unknown, that information has been discarded, requiring that assumptions be made when rebinning. Generally the assumption is that the events are evenly distributed within a histogram bin. As a result, rebinning always smooths the data. By retaining the distribution of events, one can continue using the notation of rebinning, when all that is really being done is histogramming. Figure 2 shows the ability to rebin event-based data (left) as compared to histogram data (right). As can be seen, the diffraction pattern is revealed as the bins are made smaller. With traditional histogram data, there is an effective smoothing of the data as seen in the figure. As mentioned in Section 1 , at any point data processing can continue with the histogram version of the data. In practice this can be done making a copy of the dynamic histogram. In order to efficiently histogram the data, it is best to sort the events for the histogram by time-of-flight. Then the algorithm iterates through the sorted event list, adding events to a bin, then moving on to the next bin when the next event's time-of-flight goes to a new bin. While the initial sort of $n$ events has a computational complexity of order $O(n)=n \log n$, any subsequent re-binning is performed in approximately linear time $O(n)=n$.

Histogram errors are calculated to be simply equal to the square root of the number of counts in each bin as according to Poisson statistics $[7,8]$. 


\section{Propagation of Uncertainties}

\subsection{Existing histogram mode data processing}

To provide a framework for understand event data processing, we will first describe processing histograms. A histogram can be made from event data by ignoring the wall clock time and summing together all events within histogram bin boundaries together. The term "wall clock time" refers to the absolute time of the proton pulse on target. If we sum the wall clock time and the time-of-flight, we will get the absolute time the neutron was detected. The uncertainties are just the square root of the measured intensities, $\sigma^{2}=I$, as traditionally assumed. The four types of arithmetic that one can generally do are add, subtract, multiply and divide. When doing these operations for individual histogram bins uncertainties are propagated according to traditional equations assuming there is no cross-correlation between parameters $[7,8]$.

$$
\begin{array}{ccc}
C=A+B \text { then } \sigma_{C}^{2}=\sigma_{A}^{2}+\sigma_{B}^{2} \\
C=A-B \text { then } \sigma_{C}^{2}=\sigma_{A}^{2}+\sigma_{B}^{2} \\
C=A \times B \text { then }\left(\frac{\sigma_{C}}{C}\right)^{2}=\left(\frac{\sigma_{A}}{A}\right)^{2}+\left(\frac{\sigma_{B}}{B}\right)^{2} \\
\text { or } \quad \sigma_{C}^{2}=\left(B \cdot \sigma_{A}\right)^{2}+\left(A \cdot \sigma_{B}\right)^{2} \\
C=A / B \text { then }\left(\frac{\sigma_{C}}{C}\right)^{2}=\left(\frac{\sigma_{A}}{A}\right)^{2}+\left(\frac{\sigma_{B}}{B}\right)^{2} \\
\text { or } & \sigma_{C}^{2}=\left(\frac{\sigma_{A}}{B}\right)^{2}+\left(\frac{A \cdot \sigma_{B}}{B^{2}}\right)^{2}
\end{array}
$$

\subsection{Weighted Events}

Typical data reduction steps performed on histogrammed scattering data include background subtraction, absorption corrections, normalization, and 
dividing by a reference spectrum. The discussion so far has assumed that each neutron event counted as exactly one; handling these reduction steps requires the introduction of weighted events.

A weighted event consists of a neutron event with the addition of a weight and an error (discussed in the next section). One can handle weighted events by converting un-weighted events to weighted events dynamically, only as required. Say, for instance, that an event is multiplied by a scalar, 2.0: a new weighted event will be created with a weight of 2.0 , and this will replace the original, un-weighted event. When histogramming weighted events dynamically, this event will count as two, and the resulting histogram will be the same as if the multiplication had been performed on a histogram.

The following arithmetic operations can be performed on the event data $A$ and $B$ :

$A+B:$ List of events are simply appended.

$A-B$ : List of events are appended, with $B$ 's weights set to negative.

$A \times B$ and $A / B$ : Here, the dynamic histogram of $B$ is used. The weight of each event in $A$ is multiplied/divided by the value (and error) of the histogram at the corresponding bin in the $B$ histogram. This is one occasion when the bin sizes matter.

In all four cases, the results are still lists of events, and further arithmetic can be performed with no loss of information.

\subsection{Event Data Specifics}

When using weighted events, it is necessary to propagate uncertainties in a way that is consistent with histogram methods. In essence, a list of 
events should be treated as a sparse histogram with bins of negligible width. Each of these sparse bins contains a single event $(N=1)$ and therefore its uncertainty is $\sigma=\sqrt{N}=\sqrt{1}$. There is debate as to whether or not this is an appropriate value for the uncertainties of a "small" number of counts. That is not germane to how the uncertainties are propagated $[9,10,11]$.

The methods for uncertainty propagation of histogram data, described in section 4.1, can then be applied in the usual way. With $w_{i}$ and $w_{f}$ as the initial and final weights of an event; $\sigma_{i}$ and $\sigma_{f}$ as the initial and final errors on an event:

Multiply by scalar $A: w_{f}=A \cdot w_{i} ; \sigma_{f}^{2}=A^{2} \cdot \sigma_{i}^{2}$

Multiply by scalar with error $A \pm \sigma_{A}: w_{f}=A \cdot w_{i}$ $\sigma_{f}^{2}=\left(A \cdot \sigma_{i}\right)^{2}+\left(w_{i} \cdot \sigma_{A}\right)^{2}$

Adding $N$ events into a bin : Weights are simply summed: $w_{t o t}=\sum w_{i}$. Errors are summed in quadrature: $\sigma_{\text {tot }}^{2}=\sum \sigma_{i}^{2}$

With these values, the uncertainty in a bin during on-the-fly histogramming can be performed by simply summing the square of the uncertainties of each event. For $N$ un-weighted events, $\sigma=1$, this is simply $\sqrt{N}$.

\section{Applications}

\subsection{Saving Memory}

When dealing with large numbers of events, processing speed and memory use become concerns. A way to handle this is to compress the events. This technique can be used to reduce both the number of events and their memory 

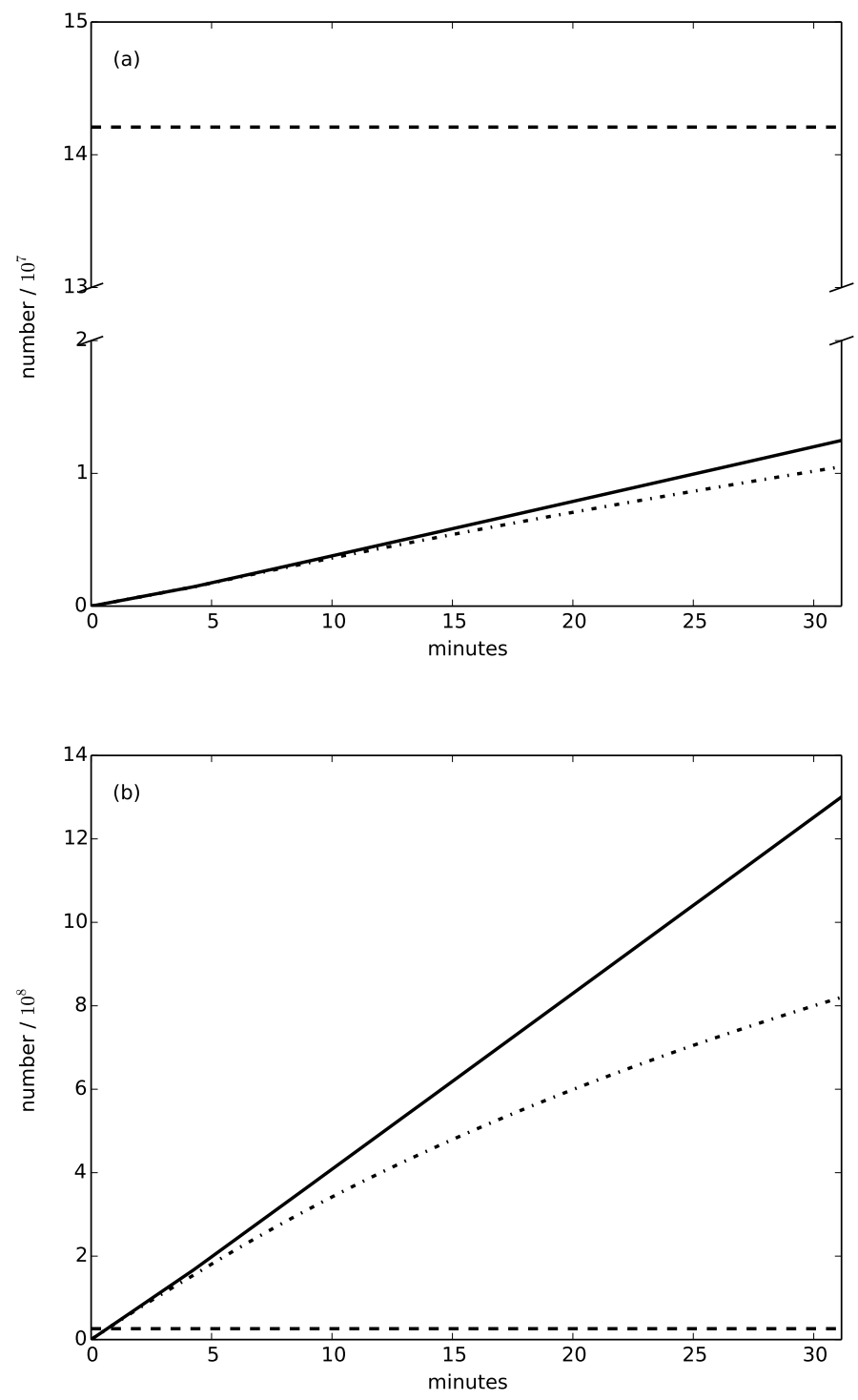

Figure 3: The data presented is from a water (in a $\mathrm{Al}$ holder) sample measured on the instrument VISION [12] separated into inelastic (a) and diffraction (b) detectors as a function of time since the measurement started. The vertical axis is in number of raw events (solid), weighted events (dot-dashed), or histogram bins (dashed). 
footprint. The algorithm iterates through the events and combines into a single weighted event any separate neutron event objects that have times-offlight within a given tolerance. As an example in time-of-flight, if 10 events have the same time-of-flight of exactly $1.000 \mathrm{~ms}$ and a weight of 1.0, they are combined into one event with a weight of 10.0: a $90 \%$ reduction in memory use. At the SNS, events' time-stamp are measured with a resolution of 100 ns; therefore, compressing events can be safely done using a tolerance of 50 ns (for example) without removing any information. In large data sets, the memory savings and speed improvements can be very significant.

There is an obvious drawback if the chosen compression tolerence is too large, then time-of-flight resolution is lost. This will leave artifacts in the data analogous to coarse binning of histogram data. A related, but separate, artifact comes from the machine precision of storing the time-of-flight, which is originally an integer number of 100ns steps, as a floating precision number. This will have a undesireable effect as the storage format will move the timeof-flight as the difference between floating point numbers and integers vary. An additional benefit of on-the-fly histogramming is reduced memory requirements for visualization. Since histograms are generated on-the-fly, they do not need to all be stored in memory at once. In many cases there is less memory needed to store the events than to store fine histograms at full instrument pixel resolution. Therefore, fine histograms that would overflow a computer's available memory can still be generated. Some experimental techniques, like single crystal diffraction, require many pixels and fine timeof-flight resolution in order to have accurate measurement of peak position and intensity. With histograms, this traditionally means a large number of 
bins. On TOPAZ $[13,14]$, a single crystal diffractometer at SNS, this would be $256 \times 256 \times 3000 \approx 2 \times 10^{8}$ for a single detector panel. Storing the events will automatically only record where there were counts which will be overwhelmingly where the Bragg peaks are. As a second example, a NeXus $[15,16]$ file from the SEQUOIA spectrometer at SNS $[17,18]$ is $265 \mathrm{MB}$ in size. The file contains approximately 5.0 million events which compresses to 4.8 million weighted events. However, with $10 \mu s$ bins, this data requires 193 million histogram bins for the time-of-flight range. Independent of storage format, this is a tremendous difference. Figure 3 shows a comparison of memory usage as a function of measurement time using the VISION instrument. While it is significantly less memory to store events than histograms for the inelastic detectors (3a), the diffraction detectors (3b) are more efficiently stored as histograms.

\subsection{Flat Background Subtraction}

We have shown above that subtracting a measured background from a given data set is straightforward. In some cases we want to subtract a constant background from the whole data set, and the value of this constant is often estimated by taking the average value over a given "flat" region of measured data. If we consider the background as a histogram, then this poses a problem if we want to subtract it from a list of events. Namely, it is impossible for us to know the distribution of events within the histogram correctly.

As a practical method to overcome this problem, our approach is to extract the list of events from the original dataset. This is then treated as a characteristic representation of the distribution of events. This region is 


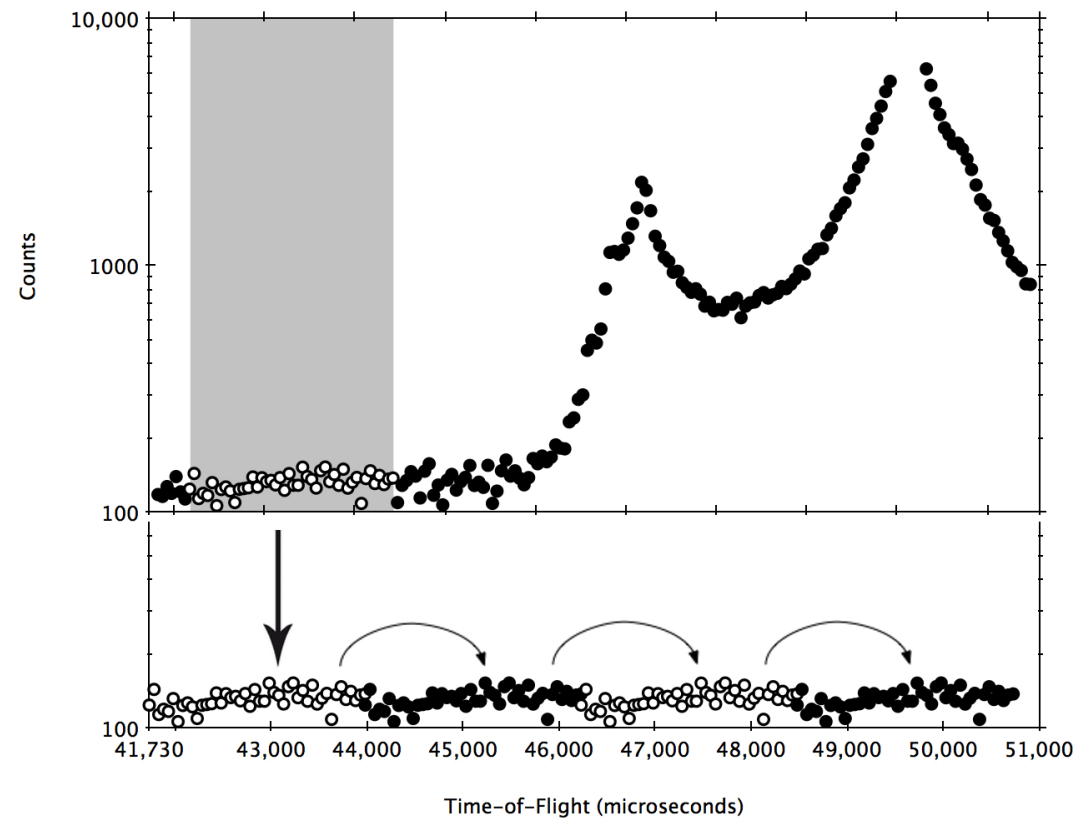

Figure 4: Schematic diagram of how we generate a flat event data set from a region of (simulated) experimental data. 
replicated and shifted until it fills up the entire data range. We now have two event lists covering the same range of data and we can simply subtract them as shown previously. Another approach would be to generate a sequence of fictitious events with a flat distribution over the entire data range.

\subsection{Filtering Events}

Since the pulse time of every event is recorded, it is possible to perform filtering on data after acquisition is complete; such filtering is impossible on histogrammed data. Filtering can reduce background noise or allow selecting parameter space more finely. Figure 5 shows a schematic of such a filtering. For filtering based on logged information there is a requirement that the clocks are all synchronized to an accuracy that is no larger than the time resolution scale of the phenomenon being observed. In practice, the resolution of commonly used time synchronization techniques [19] is adequate (e.g. 1ms upto $100 \mathrm{~ms}$ ) for "slow control" values (e.g. temperature). Filtering based on a "slow control" value is done by converting the log values to absolute times, then selecting the events based on those times[20, 21]. This can be generalized to "slicing" data where ranges of log values are used to create a list of time ranges that belong in multiple workspaces. Continuing the temperature example, slicing a measurement of temperature ramping from $100 \mathrm{~K}$ to $200 \mathrm{~K}$ in $5 \mathrm{~K}$ chunks would result in 20 event data sets from a single measurement. The experimenter can then decide whether there are sufficient statistics in each slice and increase or decrease the temperature range for the slice.

For "fast" logs which are measured at a frequency similar to the wall-clock time for the events a different technique can be used for filtering and splitting. An example of this is a magnet whose power and cooling requirements dictate 


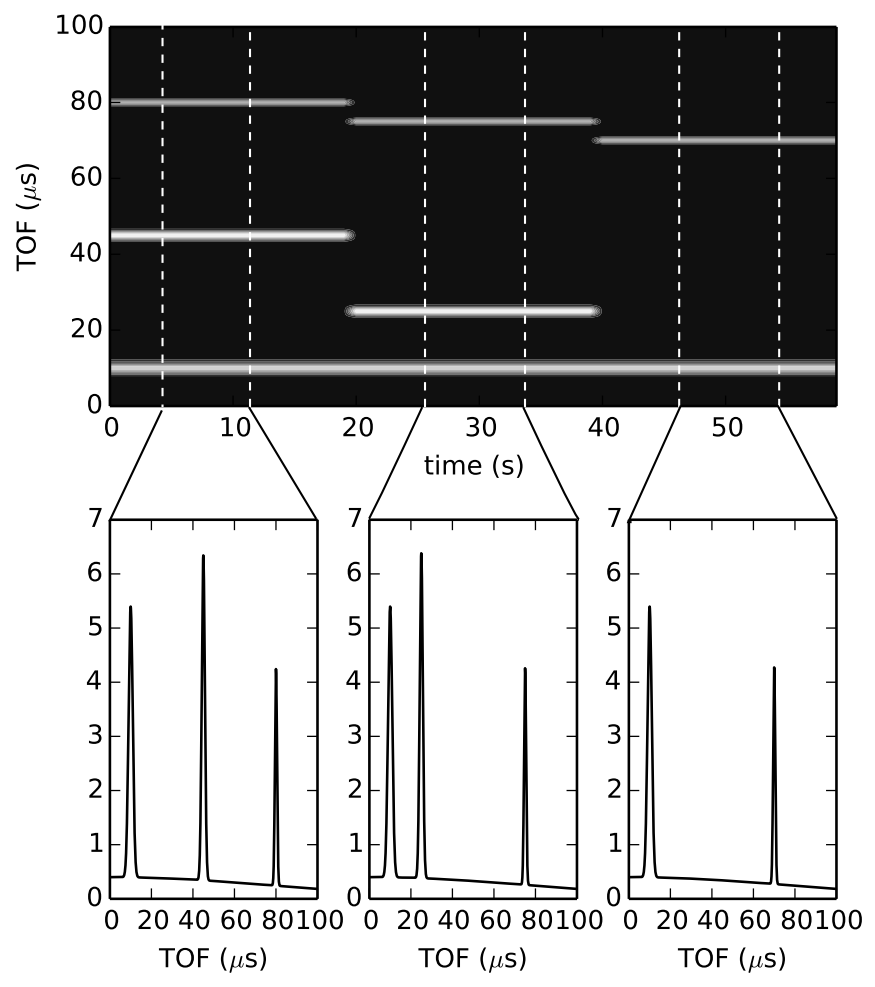

Figure 5: Schematic diagram showing event filtering capabilities. Each of the lower plots shows a wall clock integrated diffractogram of the data within the dashed lines from the upper plot. The data shown is from a simulated measurement. 
that it can only produce its field for a few microseconds then cool for several minutes. In this case, the synchronization of the times is done by the observed phenomenon. The time resolution of the log allows for frame correcting the data before filtering. The events should be filtered based on the state of the sample when the neutrons were interacting with the sample, rather than when they were at the detector. Therefore, we need to estimate how far back in time the neutron was at the sample and remove it from the events absolute time[22, 23]. The absolute time is simply the wall-clock time of the pulse added to the time-of-flight or the neutron. It is this (corrected) absolute time that should be compared to the time of the log.

\section{Summary}

We have introduced how event data can be used to process data at timeof-flight spallation neutron sources. The propagation of uncertainties for events has been shown along with some practical considerations of using this technique.

\section{Acknowledgments}

Work was sponsored by the Division of Scientific User Facilities, Office of Basic Energy Sciences, US Department of Energy, under Contract No. DE-AC05-00OR22725 with UT-Battelle, LLC. A portion of this research used data from the POWGEN and VISION instruments at Spallation Neutron Source. The authors would also like to thank Nick Draper and Timmy Ramirez-Cuesta for useful discussions. 


\section{References}

[1] Neutron News 17 (3) (2006) 3339. doi:10.1080/10448630600837556.

[2] First neutrons produced by doe's spallation neutron source, http://www .ornl.gov/ornl/news/news-releases/2006/first-neutrons-produced-by-do

[3] C. Windsor, Pulsed Neutron Scattering, Taylor and Francis, London, 1981.

[4] P. A. Egelstaff, Thermal Neutron Scattering, Vol. 8, Academic Press New York, 1965.

[5] T. G. Worlton, private communication.

[6] A. Huq, J. P. Hodges, O. Gourdon, L. Heroux, Powgen: A thirdgeneration high-resolution high-throughput powder diffraction instrument at the spallation neutron source, Zeitschrift für Kristallographie Proceedings 1 (2011) 127-135.

[7] P. R. Bevington, D. K. Robinson, Data reduction and error analysis for the physical sciences, 3rd Edition, McGraw-Hill Higher Education, 2003 .

[8] J. R. Taylor, An introduction to error analysis, 2nd Edition, University Science Books, 1997.

[9] R. Barlow, R. Cahn, G. Cowan, F. D. Lodovico, W. Ford, G. H. de Monchenault, D. Hitlin, D. Kirkby, F. L. Diberder, G. Lynch, F. Porter, S. Prell, A. Snyder, M. Sokolo, R. Waldi, Recommended 
statistical procedures for babar, Tech. Rep. 318, SLAC National Accelerator Laboratory (May 2002).

[10] J. G. Heinrich, Coverage of error bars for poisson data, Tech. Rep. 6438, University of Pennsylvania (May 2003).

[11] Error bars for poisson data, http://www.cdf.fnal.gov/physics/statistics/notes/pois_e

[12] P. A. Seeger, L. L. Daemen, J. Z. Larese, Resolution of vision, a crystalanalyzer spectrometer, Nuclear Instruments and Methods in Physics Research Section A: Accelerators, Spectrometers, Detectors and Associated Equipment 604 (3) (2009) 719728. doi:10.1016/j.nima.2009.03.204.

[13] A. J. Schultz, M. R. V. Jorgensen, X. Wang, R. L. Mikkelson, D. J. Mikkelson, V. E. Lynch, P. F. Peterson, M. L. Green, C. M. Hoffmann, Integration of neutron time-of-flight single-crystal bragg peaks in reciprocal space, J Appl Cryst 47 (3) (2014) 915921. doi:10.1107/s1600576714006372.

[14] M. R. V. Jorgensen, V. R. Hathwar, M. Sist, X. Wang, C. M. Hoffmann, A. L. Briseno, J. Overgaard, B. B. Iversen, Accurate atomic displacement parameters from time-of-flight neutron-diffraction data at topaz, Acta Cryst Sect A Found Adv 70 (6) (2014) 679681. doi:10.1107/s2053273314015599.

[15] M. Könnecke, The state of the nexus data format, Physica B: Condensed Matter 385-386 (2006) 13431345. doi:10.1016/j.physb.2006.06.106.

[16] M. Könnecke, F. A. Akeroyd, H. J. Bernstein, A. S. Brewster, S. I. Campbell, B. Clausen, S. Cottrell, J. U. Hoffmann, P. R. Jemian, 
D. Männicke, R. Osborn, P. F. Peterson, T. Richter, J. Suzuki, B. Watts, E. Wintersberger, J. Wuttke, The nexus data format, J Appl Cryst 48 (1) (2015) 301305. doi:10.1107/s1600576714027575.

[17] G. Granroth, D. Vandergriff, S. Nagler, Sequoia: A fine resolution chopper spectrometer at the sns, Physica B: Condensed Matter 385-386 (2006) 11041106. doi:10.1016/j.physb.2006.05.379.

[18] G. E. Granroth, A. I. Kolesnikov, T. E. Sherline, J. P. C. y, K. A. Ross, J. P. C. Ruff, B. D. Gaulin, S. E. Nagler, Sequoia: A newly operating chopper spectrometer at the sns, Journal of Physics: Conference Series 251 (1) (2010) 012058.

[19] The network time protocol, http://www.ntp.org.

[20] F. Weber, S. Rosenkranz, L. Pintschovius, J.-P. Castellan, R. Osborn, W. Reichardt, R. Heid, K.-P. Bohnen, E. A. Goremychkin, A. Kreyssig, et al., Physical Review Letters 109 (5). doi:10.1103/physrevlett.109.057001.

[21] P. Whitfield, A. Huq, B. Vacaliuc, P. Peterson, Extending the analysis and collection of non-ambient diffraction measurements by time and temperature-slicing of event-mode time-of-flight neutron data.

[22] K. An, H. Skorpenske, A. Stoica, D. Ma, X. Wang, E. Cakmak, First in situ lattice strains measurements under load at vulcan, Metallurgical and Materials Transactions A (2011) 1-5.

[23] H. Nojiri, S. Yoshii, M. Yasui, K. Okada, M. Matsuda, J. S. Jung, 
T. Kimura, L. Santodonato, G. E. Granroth, K. A. Ross, et al., Physical Review Letters 106 (23). doi:10.1103/physrevlett.106.237202. 